that adults who have nasal polypi have occasionally suffered from asthma, but whether this is due to obstruction or to some reflex irritation is doubtful. 2. When the occlusion of the nose occurs in infants, they are unable to suck for more than a few moments at a time, owing to the constant necessity of opening the mouth. 3. The sense of smell is abolished or blunted, owing to the impossibility of effective sniffing. 4. Expiration through the nostrils is impossible, and the flame of a candle cannot be blown out when the mouth is shut, neither can the nose be "blown" so as to expel accnmulated mucus, \&c. By holding a candle to the nose, and asking the patient to expire through the nose, we can tell which nostril is occluded when only one is thus affected. 5. The voice is altered; all nasal resonance is abolished; the tone is muffled. This mode of speech is often erroneously spoken of as "speaking through the nose."

The chief morbid conditions which may be seen by inspection of the naso-pharynx, and which may, among other symptoms, give rise to occlusion of one or both nostrils, are : 1. Catarrhal swelling of the mucous membrane, as is so commonly seen in an ordinary cold. This eatarrhal swelling, it must be remembered, occurs also in epidemic influenza, measles, and some other conditions. The secretion of the mucous membrane in this condition, varying as it does from a clear, almost limpid fluid to a thick, tenacious muco-pus, is too well known to need description. Occasionally the nose becomes the seat of a true purulent inflammation. 2. In scrofulous and syphilitic subjects nasal catarrhs are apt to become chronic; the mucous membrane generally becomes thickened and hypertrophied, or atrophied and shiny, and the secretion is apt to form crusts which stink and give rise to the name of ozoena, by which this condition is often known. 3. Abscesses of the mucous membrane occur occasionally independently of disease of the bones of the nasal cavity. 4. Syphilis very commonly attacks the nose. The presence of mucous tubercles gives rise to the "snuffles," so commonly met with in syphilitic children. Syphilitic ulcerations and infiltrations are met with here as well as elsewhere, and these conditions often lead to destruction of the cartilages, caries and necrosis of the bones, and the consequent establishment of communications between the two nostrils, or between the cavities of the nose and mouth. 5. Among the rarer causes of nasal inflammation we may mention diphtheria, erysipelas, scarlet fever, and glanders. 6. Mucous polypi may grow from any part of the nasal cavity, and vary, as you know, very greatly in size. They are usually diagnosed without difficulty, although it is well to bear in mind that other varieties of tumours, such as carcinomata and sarcomata, are found in the neighbourhood. It is well to remind you also that a hernia cerebri projecting into the nasal cavity through a congenital fissure in the base of the skull has been mistaken for a polypus. 7. It is only necessary to mention the fact that foreign bodies, such as peas and lumbrici, are occasionally found in the nose.

A very common symptom of disease of the naso-pharynx is impairment of hearing, owing to the mechanical occlusion of the Eustachian tubes. When a patient complains of being deaf, you should always examine the pharynx.

\section{INSANITY; ITS TREATMENT AND PREVENTION.}

The Presidential Address for 1880 of the Border Branch of the British Medical Association.

By J. A. CAMPBELL, M.D., F.R.S. EDIN., MEDICIL SUPERINTENDENT, GARLANDS ASYLUM, CARLISLE. (Concluded from p. 999.)

Ix talling over the matter of the causation of insanity with meinbers of the profession in asylum practice, I have always strongly expressed my disbelief in purely mental causes having an intluence to the extent generally supposed in producing this disease, and $\mathrm{I}$ have been frequently answered that my views arose from my principally dealing with cases in the lesselucated grades of society, and that among the educated and in the higher ranks purely mental causes were far nore common. I still, however, adhere to my views, and think that cases are frequently supposed to be caused by extra study, mental anxiety, orerwork, religious doubts, when, in reality, other agencies, habits, and vices have been the real cause of all the mischief.

I have already placed before you the difficulties one ex. periences in arriving with anything like absolute certainty at the cause of disease. In several cases of clergymen, clerks, travellers, \&c., I have, on careful examination, found that where all at first pointed to a mental causation, fuller inquiry showed a physical. I am quite aware that a few general re marks or isolated cases go for nothing in dealing with such a subject. I therefore submit the following.

I have most carefully examined into the causation of the cases sent to Garlands Asylum during the past two years. In this period 238 patients were admitted : in 153 the malady was distinctly due to physical causes ; in 36 only to moral causes. I have already pointed out that exception might be taken to results arrived at in an asylum whose population is principally derived from an agricultural, or at any rate a manual, self supporting source. Let us, then, look for a moment at a likely cause of insanity-mental shock or worry from money losses. One would imagine that few things could have a more deleterious effect on the human mind than losses which keep one tottering on the brink, or that cause distinct and total ruin at once. Thrilling tales are founded on such occurrences, and our profession occasionally record wonderful cases, the result of unexpected and sudden ruin or reverses in fortune, cases of death from shock, insanity, paralysis, sudden ageing, change in colour of hair, \&c. Now one of the greatest conmercial casualties which of late years has affected Scotland occurred by the stoppage of the City of Glasgow Bank on the 2nd of October, 1878. This failure caused very great distress to many, ruin to some, and most undoubtedly anxiety of a severe nature to all concerned with it directly, and to many others indirectly. From the report of the liquidators, ${ }^{1}$ I find that there were 1093 shareholders in their own richts, 100 life renters, 192 bodies of trustees, 108 persons whose wives held stock, 34 shareholders as trustees; the total number of separate persons entered on the list was 1819; 599 surrendered their estates, unable to pay the first call made upon them ; 354 who met the first call were unable to pay the second. I made an inquiry from the medical officers of seven royal asylums, two private, ten district, and two parochial, to find out how many patients up to the time of my inquiry (April, 1880) had been sent to asylums from this cause. I found that six patients were admitted in whom the disease was considered to be directly caused by this bank failure, while in two it was considered the indirect cause. In Garlands, in 1879, seventeen cases were sent in from excessive drinking, and these from two counties in one year. I do not for one moment doubt that many cases have been evilly acted upon by this failure; probably cases of insanity have occurred which did not require asylum treatment. I am only dealing with this failure as a factor in causing insanity, which required asylum treatment; and the result of my inquiry shows that a bank failure of a magnitude which allows one to call it a national calamity, which directly affected 1819 persons-and who knows how many indirectly?-which must have produced an appalling amount of mental shock, an immeasurable amount of anxiety and worry, and an as yet unknown amount of hardship, only sent eight persons to the twenty-one principal Scotch asylums. This should lighten the minds of directors.

During 1876 and 1878 the Commissioners in Lunacy, with the co-operation of the medical officers of asylums, collected and tabulated certain data, among others the causes of the insanity (arrived at after careful scrutiny and consideration of each case individually on the part of the asylum medical officers), with regard to the admissions which took place into English asylums. I consider that the return for 1878 is the most accurate of the sort that we have ever had access to, and I therefore refer to it. 13,309 were admitted into English asylums during 1878. Moral causes as the excitant or predisposer were considered to have caused insanity in 3199 cases; domestic trouble, adverse circumstances, mental anxiety, overwork, religious excitement, love affairs, fright, and nervous shock-24 per cent. of the admissions nuly were caused by such agencies as I have mentioned acting directly on the mind.

When we turn to the physical causes we find $641 \mathrm{~s}$ cases accounted for by them, 48 per cent. of the whole admis. sions being attributable to direct physical causes, such as intemperance in drink, or sexual and venereal disease.

I hare to thank one of the liquidators for kindly furnishing me with 
To doubt some of the physical causes are distinctly nonpreventable, such as old age; but many of them are, and it is satisfactory to have something tangible, recognisable, curable. and preventable to go on. Taking intemperance in drink, it is put down as the exciting cause of insanity in 1351 of the admissions, the predisposing in 415 -altogether it is credited with causing 14.6 per cent. of the whole admissions. Lactation as a predisposing cause affected 15 , as an exciting 101 ; it caused 1.9 , nearly 2 per cent., of all the female admissions.

Education, elevation of the mental faculties, a diffusion of knowledge as to the requirements of the bodily health, an accuirement of desires for rational amusements and intellectual relaxations, among the mass, have for long been pointed ont as the preventive for insanity caused by giving rein to the baser passions and appetites. As this is long in coming, we must look for, hope for, urge as far as we can, and point out, as we as a profession have done, and are constantly doing, stringent and easily enforced legislation which will deal with habitual drunkenness, prevent the adulteration of malt and spirituous liquors, and the sale of newly made spirits which contain constituents especially deleterious to brain-tissue. Such legislation must be enforced to be of value ; permissive legislation on such a subject is valueless.

Are doctors sufficiently consulted by heads of families either as regards bodily or mental health -in fact, as regards the prevention of disease in their offspring? Are they not in the majority of cases merely called in to attempt to cure a disease which has been long going on, and which might have been prevented?

With regard to children, I believe medical men are now more consulted than they used to be about their proper mutrition in infancy and childhood; but is sufficient attention paid to their education, recreations, the choice of a profession, the capacity of the mind for severe mental toil, the question of bodily disease, its development under certain conditions and in certain occupations, and its power of being retarded or prevented by constant attention to bodily health and freedom from mental strain? Are such subjects properly present before the minds of parents, guardians, and the medical profession at large? This is a difficult subject, and each year it is becoming more and more pressing; and when we look at the causation of many cases of mental disease we have the conviction forced upon us that had such and such a case been in different circumstances, it would have been most unlikely that he would have become insane.

Then, leaving the period of childhood and coming to that of youth and budding manhood and womanhood, should not the primary laws of physiology be taught? Would it not be well for those who hope to live for the allotted span in this world to know how they live, and how best to live to keep living! and some truths, of course in a guarded way, should be communicated to them by their elders about their reproductive systems, which to many, especially those of a neurotic family, is the cause of many uneasy feelings, wonderments, and doubts. From my experience, I am quite sure thatin many individuals of both sexes evil practices and habits have often arisen in ignorance-habits such as masturbation, witis its train of evil consequences to mind and body, excitement, vacillation, depraved inclinations of all sorts, in the male; and similar, if less marked, mental changes, with hysteria predominating, in the female, an unhappy ruined life and weakened body, at the slightest, and in extreme cases existence in an asylum, a trouble and a care to all, for in such cases, at their worst, impulsiveness is a prominent feature, and dangerous outbreaks of short, almost momentary duration alternate with depression. Such persons are usually filthy in their habits, and nearly always end in complete dementia, frequently run their bodily health down to the lowest ebb, and die of phthisis.

Then, passing onwards, is marriage at present looked upon in its proper light? Are the prospects of a healthy progeny considered? Wealth, social position, even creed, are duly thrught of - why not mental and physical properties? Each vear nne hears of marriages broken off for monetary or scinl reasons, but is the family history of the young couple ever considered-the great probabilities of disease in one or toth, the family home broken up by puerperal mania, the children descended from two insane or phthisically-inclined frarents, causing anxiety, discomfort, grief, and useless rerret, instead of happiness, bright anticipations, and increace of love ; and when, by eating the fruit, the knowledge $i=$ gained to see that all this could have been prevented, that knowledge acquired in time might have enabled one to guide their selection, and to influence their love, as well in matters of this sort as in those of monetary and social consequence.

It would almost seem as if there was an elective affinity between persons with a neurotic taint, especially those descended from suicidally disposed ancestors. I have often been led to notice how frequently they intermarry. They are often in the blush of youth well-developed and goodlooking, arrive more quickly at maturity than their more healthily constituted compeers, have more adaptation of character, are more subject to the emotions, and probably being more deficient in the sterling qualities required in life's journey, yield themselves with more abandon to the pleasures of the moment, without a care for the morrow. I think that in this probably lies the fascination often noticed in such cases as I describe. But while the state I have presented occurs in some instances, and at the early periods of life, there is another side to be heard, and even the individuals who while they are young present cheerful aspects often get morbidly dull in the middle and later periods of life. There are many neurotically descended who feel their burden from the first, and feel it more so from their surroundings. To be descended from insane or even peculiar and eccentric ancestors is a great hardship, but to have to live with them and be surrounded with a large circle of similarly constituted relatives is nearly as bad, and undoubtedly has a bad influence on the young. That people unintentionally acquire peculiarities from associates is well known ; that a dread of an outbreak of nervous disease is often present in the minds of those who inherit strong neurotic taint is also well known; and that this dread may be almost extinguished, or kept constantly on the alert by the surroundings, stands to reason. Persons with strong neurotic taint should, for their own safety and happiness, live as much as they can amongst healthily constituted strangers. They should, as far as possible, keep clear of the associations which constantly bring their unpleasant inheritance before them prominently. In many cases a change of county, or even of country, is really a great safeguard. This should be known.

Only when such evils take place as puerperal mania is the advice of the medical man asked for or listened to as to the probability of a recurrence of the malady at the next confinement, or as to the time which ought to be allowed to elapse before the woman is again subjected to the dangers of another attack. After a severe attack of puerperal insanity the nervous system must take a long time to recover thoroughly; and though the patient may ostensibly be well, and not need asylum or other treatment, yet I believe that a considerable period without any extra calls on the system generally is necessary for total and complete recovery. I always tell husbands my views in this respect when $I$ discharge patients who have recovered from puerperal mania, and advise for their own comfort that a year should elapse before another pregnancy; but usually such advice is un. attended to, and a quickly following confinement brings a recurrence of the insanity. The most marked case that has lately come under my notice was E. J-. Father drowned himself. She had been always rather nervous. Came in on May 15th, 1877, labouring under puerperal mania. This was two weeks after confinement. She was excited for a long time, but gradually got better, and was discharged on March 1lth, 1878. I expressed myself strongly to the husband, pointing ont that in this case, from the family history, it was even more essential than in ordinary cases that a considerable interval should elapse before the next confinement. This patient was readmitted, pregnant, on Sept. 21st, 1878. She continued in a state of excitement during her pregnancy, and was confined of an apparently healthy male child on Dec. 15th, 1878, 288 days from her discharge, so that she probably became pregnant within eight days of leaving. She is still in the asylum, an excited, hysterical female, at present entirely devoid of self-control. Had she not become pregnant, I think that in all probability she would have retained her reason.

In nearly all the cases of insanity from over-nursing that come under my observation the patient has been nursing up to the time of being brought to the asylum. In the majority of cases, after the milk has been got rid of by rubbing the breasts with a saturated solution of extract of belladonna in glycerine and the administration of a saline aperient, and the patient afterwards treated with iron and tonics, recovery is very speedy, if the patients were warned about such an 
occurrence as nursing proving too much for them, if the too great drain on the system were prevented by partially feeding the child or entirely ceasing nursing when the bodily health was seen to suffer and the patient was becoming dull, the attack might be averted. I have always thought that medical advice was too tardily sought in such cases.

The time between gestations, even when not followed by immediate insanity, does not meet with the consideration it merits. I frequently see cases of mental disease, in many complicated with phthisis, in which numerous and rapidly following pregnancies have undoubtedly been the cause; and in a considerable number of cases of middle-aged and oldish women, with lowered vitality, broken-down constitutions, absence of nervous power, and consequent dementia or melancholia, I have, on scrutiny, clearly realised that unduly rapid pregnancies or miscarriages have been the cause of such a state. And yet in cattle this is avoided; a breeder of good stock, always after a miscarriage, does not allow another pregnancy till the time he would have done so had the female gone the full time.

That fright, nervous shock, illnesses, and mental anxieties exercise in some manner a direct influence on the unborn offspring through the mother is well understood. Maternal influences during the period of gestation is the one subject of this olass which has received most attention, and great care is usually expended on the interesting patient. Probably the laws of some of the nations who did away with weakly offspring gave the initial impetus to this attention, but I doubt whether much family happiness would not result by attention to the life, health, and habits of would-be parents during the period immediately preceding gestation. It is well known that frequently children procreated during alcoholic excess are imbecile or hydrocephalic. Deviations from health, both mental and physical, may be a question of degree. One frequently sees the children of intemperate parents stupid, dull, and coarsely formed. I have no doubt the mental state of the parents during the period immediately preceding procreation has a great influence on the future child. I should like to hear, as some day we shall, something as to the effects of the siege of Paris and the Franco-German war on the children procreated in Paris during that eventful period. In Jewish history newly married men were exempt from martial service.

I trust that the portion of my address which deals with the treatment of insanity will convince you that practitioners in this department, as in the others of our profession, have at heart not only the good treatment of the patients, but also the advancement of our knowledge of the subject.

As regards the prevention of insanity, all our profession are interested in it. I have brought before you the thoughts which have occurred to my mind as worthy of your consideration. Ours is a progressive study; it should end only with our lives. As each year rolls past we should have observed some new facts, made ourselves more acquainted with the wonderful laws of nature which have specially to deal with humanity, and fitted ourselves to advise with such certainty as is to be expected from fallible man on many of the points upon which I have touched, for each year more will be expected from us in this direction.

\section{HUMAN AND ANIMAL VARIOL E : A STUDY IN COMPARATIVE PATHOLOGY.}

\section{BY GEOR GE FLEMING, F.R.C.V.S., ARMY VETERINARY INSPECTOR. \\ (Continued from vol. i., page 834.)}

I HAVE already alluded to the results of small-pox inoculations on the horse, and shown that, as on the ox, they were either negative or nearly so, the positive results yielding only the most trifling evidence of infection, and nothing at all like horse-pox being ever produced from the insertion of the small-pox virus into the skin of the horse, while retransmission to mankind only gave rise to small-pox. On the contrary, as we have seen, horse-pox inoculated on the cow produces what in every way corresponds to cow-pox, or on man to vaccinia. From the cow and man horse-pox can be transmitted indefinitely, and while its action is modified by repeated transmissions, its protective influence against variola is undiminished. This has been demon. strated times almost without number, from the days of Jenner up to the present date. The most interesting and instructive of recent demonstrations in this direction is that recorded by Dr. Pingaud, in a communication presented to the Paris Academy of Medicine a few months ago. Having observed, with Drs. Viseux and Thomas, an epizooty of horse-pox in February, 1879, he determined to make some experiments, and selected as a vacciniferous subject a fouryear-old horse affected with the disease, but whose ante. cedents were well known, and whose health at other times was excellent. The cutaneous eruption was discrete ; there were only a few crusts of pustules about the hollow of the pasterns ; but in the mouth, and within the upper lip, the mucous membrane was studded with vesicles offering the characteristic nacrous aspect. Lymph was collected from these pustules with the greatest care, and seven young soldiers of the 10th Hussars, who had not been vaccinated, were inoculated. On the sixth day six of the men showed at the seat of inoculation the characteristic vaccine vesicles, which had a somewhat inflamed base. From four of the soldiers lymph was taken; with this sixty-four men -eight of whom had not been vaccinated--were inoculated. In forty of these the result was positive-60 per cent. successful vaccinations. With none of the patients were there any serious inflammatory symptoms, and only in a very small number did the inoculations assume a furunculous aspect. Calves were inoculated from the horse, but the pro. portion of successes was only 48 per cent., rather going to prove that the virus became weakened in the calves.

I have stated that horse-pox and cow-pox are almost, if not quite identical in their effects, when transmitted by inoculation to man, the ox, or the horse. Chauveau inocu. lated five horses and two asses with animal vaccine from Naples; the youngest animal was seven years old, the others from sixteen to twenty years of age. For five and six days there were no signs, but in from five to eight days the seat of puncture became markedly papulous, the papules increasing in size until about the tenth day, when they were acuminated, had a very broad base, and were red, painful, and hard. From the ninth to the twelfth day was the period of secretion, the epidermis becoming slightly raised throughout the whole extent of the papule by an exudation of limpid citron-coloured serum; this dried into yellowish transparent crusts, very different in appearance from those of vaccinia in man or cow. The secretion from the lymph continued for several days, and ceased from the thirteenth to the seventeenth day. When the crust was removed there appeared a moist granular red surface level with the skin, but having a deep central cavity, a kind of umbilicus, in which had been fixed, like the head of a nail, the prominent under-surface of the crust. There was no febrile reaction. In the asses there was shedding of the hair and epidermis in various parts, with abundant serous exudation. As com. pared with its evolution in man and the cow, the vesicle or pustule was slower, and there was a difference in its shape and character, the pustule being acuminated, the lymph scanty, and the umbilicus small or altogether absent; thongh in an ass sixteen years old the pustule had from the commencement a central umbilicus. In the horse the crust extends over all the pustule, while in man and the cow only the centre is covered. In the ox the lymph is rarely abun dant, and there is never anything more than a thin brown crust; but in the horse the crust is wide, thick, rough on the surface, and transparent and citrine-tinted.

\section{SHEEP-POX}

Sheep-pox differs from cow- and horse-pox in having a history extending over some centuries, and in being closely allied to small-pox in its extension, this depending not only on its contagious but also on its infectious properties, and the eruption being more or less general over the body. Indeed, so far as intensity of virulence is concerned, its appearing in an epizootic form, as well as the serious symptoms and nortality accompanying sheep-pox, there is the closest resenblance between it and humar variola. We have seen that cow-pox and horse-pox are far from being serious disorders, the affected animals, in the majority of instances, being 